\title{
Landau Velocity for Collective Quantum Hall Breakdown in Bilayer Graphene
}

\author{
W. Yang, ${ }^{1}$ H. Graef, ${ }^{1,2,6}$ X. Lu, ${ }^{3}$ G. Zhang, ${ }^{3}$ T. Taniguchi, ${ }^{4}$ K. Watanabe, ${ }^{4}$ A. Bachtold, ${ }^{5}$ E. H. T. Teo, ${ }^{6}$ E. Baudin, ${ }^{1}$ \\ E. Bocquillon, ${ }^{1}$ G. Fève, ${ }^{1}$ J-M. Berroir, ${ }^{1}$ D. Carpentier, ${ }^{7}$ M. O. Goerbig, ${ }^{8}$ and B. Plaçais ${ }^{1, *}$ \\ ${ }^{1}$ Laboratoire Pierre Aigrain, Ecole Normale Supérieure, PSL University, Sorbonne Université, \\ Université Paris Diderot, Sorbonne Paris Cité, CNRS, 24 rue Lhomond, 75005 Paris, France \\ ${ }^{2}$ CINTRA, UMI 3288, CNRS/NTU/Thales, Research Techno Plaza, 50 Nanyang Drive, Singapore 637553 Singapore \\ ${ }^{3}$ Beijing National Laboratory for Condensed Matter Physics and Institute of Physics, \\ Chinese Academy of Sciences, Beijing 100190, China \\ ${ }^{4}$ Advanced Materials Laboratory, National Institute for Materials Science, Tsukuba, Ibaraki 305-0047, Japan \\ ${ }^{5}$ ICFO-Institut de Ciencies Fotoniques, The Barcelona Institute of Science and Technology, \\ 08860 Castelldefels, Barcelona, Spain \\ ${ }^{6}$ Nanyang Technological University, School of Electrical and Electronic Engineering, \\ 50 Nanyang Ave, Singapore 639798, Singapore \\ ${ }^{7}$ University of Lyon, ENS de Lyon, University Claude Bernard, CNRS, Laboratoire de Physique, Lyon F-69342, France \\ ${ }^{8}$ Laboratoire de Physique des Solides, CNRS UMR 8502, University Paris-Sud, Université Paris-Saclay, \\ F-91405 Orsay Cedex, France
}

(Received 28 May 2018; published 25 September 2018)

\begin{abstract}
Breakdown of the quantum Hall effect (QHE) is commonly associated with an electric field approaching the inter-Landau-level (LL) Zener field, the ratio of the Landau gap and the cyclotron radius. Eluded in semiconducting heterostructures, in spite of extensive investigation, the intrinsic Zener limit is reported here using high-mobility bilayer graphene and high-frequency current noise. We show that collective excitations arising from electron-electron interactions are essential. Beyond a noiseless ballistic QHE regime a large super-Poissonian shot noise signals the breakdown via inter-LL scattering. The breakdown is ultimately limited by collective excitations in a regime where phonon and impurity scattering are quenched. The breakdown mechanism can be described by a Landau critical velocity as it bears strong similarities with the roton mechanism of superfluids. In addition, we show that breakdown is a precursor of an electricfield induced QHE-metal transition.
\end{abstract}

DOI: 10.1103/PhysRevLett.121.136804

The Fermi sea of a 2D electronic system (2DES) is unstable at high magnetic field ( $B$ field) toward the formation of discrete Landau levels, giving rise to the quantum Hall effect (QHE) [1], where the bulk is a quantum Hall insulator. The QHE has led to many developments in metrology [2,3] and quantum electronics [4]. The fate of the QHE at high electric field ( $E$ field) remains an open question, as well as the nature of the phase reached when the drift velocity $\mathbf{v}_{D}=\mathbf{E} \times \mathbf{B} / B^{2}$ approaches the cyclotron velocity $R_{c} \omega_{c}$, where $R_{c}$ and $\omega_{c}$ are the cyclotron radius and frequency, resulting in cyclotron orbit breaking. A precursor of the transition is the quantum Hall breakdown reported soon after the discovery of QHE [5-7]. The breakdown field $E_{\mathrm{bd}}$ marks the onset of longitudinal resistance and dissipation. Its natural scale is the Zener field, $E_{c} \sim \hbar \omega_{c} / e R_{c}$, with $\omega_{c}=e B / m^{*}$ and $R_{c} \sim \sqrt{N} l_{B}$, where $m^{*}$ is the effective mass, $N$ the number of occupied Landau levels (LLs), and $l_{B}=\sqrt{\hbar / e B}$ the magnetic length [8]. The relevance of the Zener mechanism was soon questioned as $E_{c}$ exceeds experimental $E_{\mathrm{bd}}$. Besides, genuine inter-LL tunneling suffers from a strong momentum mismatch at finite doping, $\Delta k=2 k_{F}$, where $k_{F}=\sqrt{2 N} / l_{B}$ is the Fermi wave vector [9]. It can be circumvented assuming impurity-assisted or phononmediated quasielastic inter-LL scattering (QUILLS) $[8,10]$.

Breakdown was extensively investigated in semiconductors [6,7,11-16] and graphene [17-20], but mainly in Hall bars. Few experiments used constrictions [7,11], or Corbino geometries [21-24], with the purpose of achieving homogeneous current, or $E$-field, distributions. In all cases $E_{\mathrm{bd}}<E_{c}$ and critical Hall current densities $J \lesssim$ $50 \mathrm{~A} / \mathrm{m}$ [18]. The leading explanation thus shifted to a thermal instability, driven by the imbalance between dissipation and phonon relaxation [25]; its threshold is smaller and material dependent [17,18,26,27]. According to low-frequency noise measurements, the thermal instability eventually gives rise to electron avalanches $[11,21,22]$. On comparing breakdown $\left(v_{\mathrm{bd}}=J_{\mathrm{bd}} / n e\right)$ and Zener $\left(v_{c}=E_{c} / B\right)$ velocities one finds $v_{\text {bd }} / v_{c} \sim$ 0.01 in Hall bars and $v_{\mathrm{bd}} / v_{c} \lesssim 0.5$ in constrictions, presumably due to electrostatic inhomogeneities.

We propose a scenario where breakdown stems from the spontaneous generation of collective excitations at the 

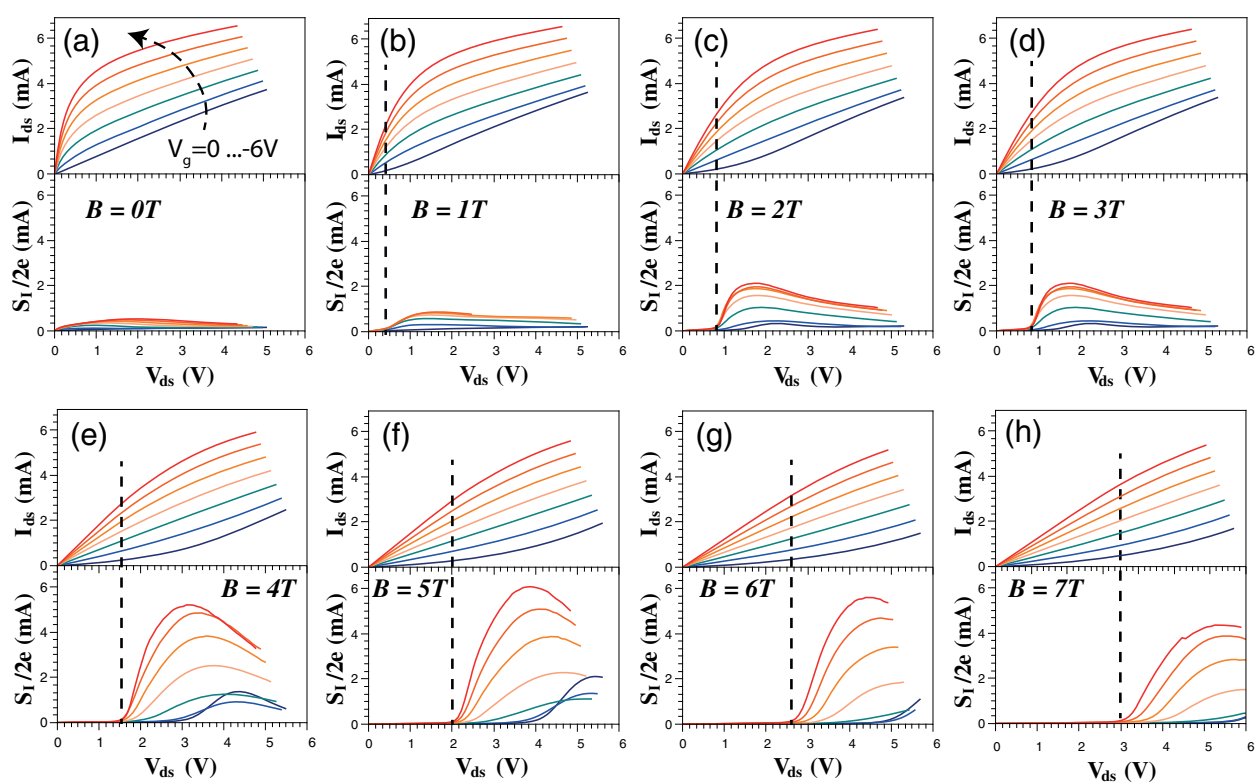

FIG. 1. High electric-field magnetostransport and $5 \mathrm{GHz}$ shot noise in bilayer graphene of dimensions $L \times W=4 \times 3 \mu \mathrm{m}$ at $T=4 \mathrm{~K}$. (a)-(h) High-bias transport current $I_{\mathrm{ds}}\left(V_{\mathrm{ds}}\right)$ and shot-noise current $S_{I} / 2 e\left(V_{\mathrm{ds}}\right)$ in the hole doping QHE regime $|n|=0-4.3 \times 10^{12} \mathrm{~cm}^{-2}$ corresponding to $V_{g}=0 \rightarrow-6 \mathrm{~V}$. In quantizing fields $(B \gtrsim 2 \mathrm{~T})$ shot noise is suppressed at low bias where $G_{\mathrm{ds}}=|n| e / B$, rises up abruptly at a breakdown voltage (dashed black lines), peaks to a large value eventually reaching the full shot-noise limit $S_{I} \approx 2 e I_{\mathrm{ds}}$ $(B=5 \mathrm{~T})$, and vanishes as conductance saturates to a field-independent value $G_{\text {sat }} \simeq 0.6 \mathrm{mS}$.

magnetoexciton (ME) minimum [28]. Similar excitations exist in fractional QHE [29] that are longitudinal and called magnetorotons in reference to the roton minimum in superfluids [30]. Elusive in spectroscopy, high-momentum excitations can be probed by measuring the critical drift velocity (Landau velocity $v_{L}$ ), when their excitation gap, $\omega(q)-v_{L} q$ in the laboratory frame of reference, vanishes. Recent examples are given by superfluid helium-3 [31] and quantum gases [32]. The case of MEs is generically similar with, however, a transverse polarization, and a series of $N$ minima in the dispersion relation with a spectral weight vanishing for $q \gtrsim k_{F}$ [33]. With $q_{\mathrm{ME}} \sim k_{F}$ and $\omega\left(q_{\mathrm{ME}}\right) \sim \omega_{c}$, MEs yield $v_{L} \sim \omega_{c} / k_{F}$ identical to the Zener velocity $v_{c}=E_{c} / B \sim \omega_{c} l_{B} / \sqrt{N}$. We note that a similar mechanism was proposed in Ref. [40] to explain the steplike breakdown in $\mathrm{GaAs} / \mathrm{AlGaAs}$ heterostructures [6,41]. We report on QHE measurements in a clean bilayer graphene (BLG) microsample, a two-terminal rectangular sample of dimensions $L \times W=4 \times 3 \mu \mathrm{m}$ [33], chosen here as a prototypal massive 2DES $\left(m^{*} / m_{0} \simeq 0.03\right)$. The QHE regime persists up to large velocities, $v_{\text {bd }} \lesssim 1.7 \times 10^{5} \mathrm{~m} / \mathrm{s}$ [Fig. 2(a)], approaching the intrinsic limit $v_{\text {bd }} \simeq v_{c}$. We reveal the collective nature of breakdown using shot noise. We report on large Fano factors $\mathcal{F} \lesssim 20$ and highlight the scaling of shot noise with the Zener field and Landau energy.

A representative set of the current and noise measurements is presented in Figs. 1(a)-1(h). The bias-induced QHE breakdown is signaled by a strong uprise of noise, widely exceeding the zero $B$-field value, and eventually approaching a full shot noise limit $S_{I} \approx 2 e I_{\mathrm{ds}}$. The contrast with the ballistic QHE regime, where noise is essentially suppressed [42], is striking with a peak noise $\left(E \lesssim 2 E_{\mathrm{bd}}\right)$ highlighting a tumultuous breakdown. The onset of noise provides an unambiguous determination of breakdown, because it signals the departure from ballistic transport associated to well-defined LLs. The breakdown voltage $V_{\mathrm{bd}}(B)=E_{\mathrm{bd}} W$ is indicated by dashed lines in Figs. 1(a)-1(h). As seen in the figure, it corresponds to a deviation from the dissipationless Hall transport regime $I_{\mathrm{ds}}=G_{H} V_{\mathrm{ds}}$ with $G_{H}=|n| e / B$ [43]. In this sample the breakdown currents, $J_{\text {bd }} \simeq 1200 \mathrm{~A} / \mathrm{m}$ $\left(B=7 \mathrm{~T}, V_{g}=-6 \mathrm{~V}\right)$, exceed previously reported values $\left(J_{\mathrm{bd}} \lesssim 50 \mathrm{~A} / \mathrm{m}\right)$. The decrease of noise at $E \gg E_{\mathrm{bd}}$ is different in nature; it signals the ignition of energy relaxation restricting the electronic temperature $k_{B} T_{e} \simeq S_{I} / 4 G_{\mathrm{ds}}$ below a hot-electron limit $k_{B} T_{e} \sim e V_{\mathrm{ds}} / 2$, indicating the recovery of a metallic behavior. Conversely, the fact that a full shot noise can be reached at intermediate bias is direct evidence of the quenching of phonon relaxation mechanisms. This observation is consistent with the $q=2 k_{F}$ resonant electron-phonon coupling reported in phononinduced resistance oscillations [9], implying a suppression of conventional phonon relaxation mechanisms at low temperature and high electron density, i.e., in the BlochGrüneisen (BG) regime [44]. It also excludes significant contribution from impurity-assisted supercollisions [45], intrinsic optical phonon cooling [46], and demonstrates in situ the quenching of hyperbolic phonon cooling under quantizing magnetic fields [37]. Importantly for the interpretation of breakdown, this observation rules out the 

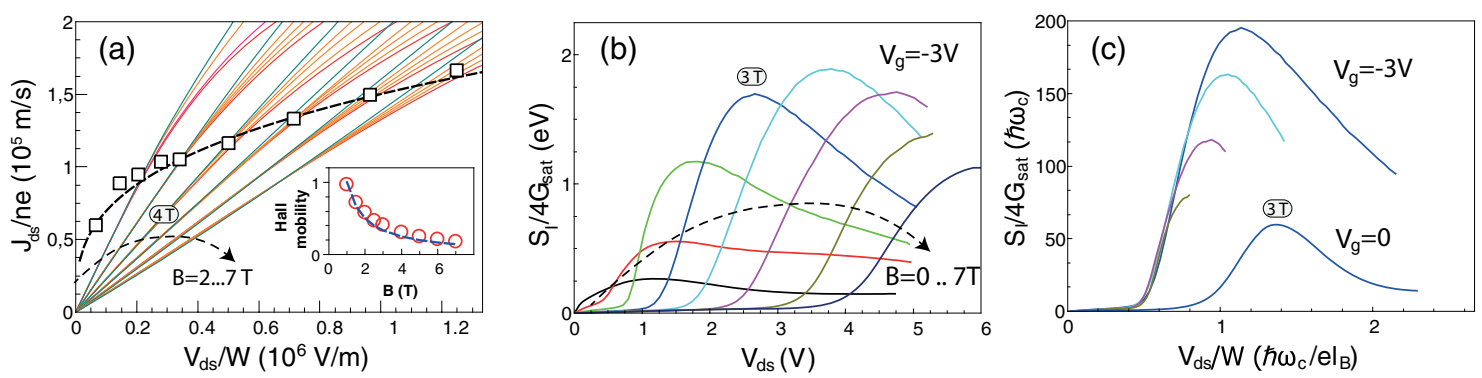

FIG. 2. Scaling behavior of current and noise in the QHE regime. (a) Bias dependence of the drift velocity $v_{D}=J_{\mathrm{ds}} /|n| e$ deduced from current data in Fig. 1. The $B=2 \rightarrow 7 \mathrm{~T}$ data of different densities bunch in the QHE regime and stray above the breakdown field. The slopes, represented as a Hall mobility in the inset, obey the expected law $\mu=1 / B$ (blue dashed line in $\mathrm{m}^{2} / \mathrm{Vs}$ ). Black squares correspond to a typical breakdown voltage deduced from the onset of noise [dashed lines in Figs. 1(a)-1(h)]. They can be fitted to the Zener tunneling limit $v_{c}=\left(E_{c} \hbar e / N m^{* 2}\right)^{1 / 3}$ (black dashed line) by taking a constant landau level $N=5$ corresponding to the median experimental value [dashed yellow line in Figs. 1(b)]. (b) Bias dependence of $|n|=2.15 \times 10^{12} \mathrm{~cm}^{-2}$ shot-noise data [from Figs. 1(a)-1(h)] in increasing magnetic fields $B=0 \rightarrow 7 \mathrm{~T}$. Ballistic transport is characterized by a suppression of noise below a critical voltage ranging from $V_{c} \simeq 0.6$ (at $B=2$ ) to $V_{c} \simeq 4 \mathrm{~V}$ (at $B=7 \mathrm{~T}$ ). Current noise is scaled by the constant saturation conductance $G_{\text {sat }}=0.6 \mathrm{mS}$ to be expressed as a noise temperature. (c) The above noise data obey a quantum Hall scaling at breakdown where Hall voltage and noise temperature are scaled to the maximum Zener field $E_{c}=\hbar \omega_{c} / e l_{B}$ and cyclotron energy $\hbar \omega_{c}$, respectively.

thermal scenario of Refs. [18,26,27] or phonon-assisted QUILLS in our experiment, which is performed below the BG temperature. The fact that supercollisions are not seen at zero field in Ref. [37] is a strong indication that impurityassisted QUILLS should be very weak. Finally, another feature of transport in Figs. 1(a)-1(h) is the saturation of the differential conductance at high bias to a $G_{\text {sat }} \simeq 0.6 \mathrm{mS}$ that is independent of doping and magnetic field, suggesting that transport becomes metallic.

Signatures of QHE breakdown in transport are best captured by the drift velocity $v_{D}=J_{\mathrm{ds}} / n e$ as function of the Hall field $E=V_{\mathrm{ds}} / W$ in Fig. 2(a). It shows a universal Hall mobility, pictured by the bunching of constant $B$-field data along $v_{D}=E / B=\mu E$ lines. Breakdown is signaled by fanning out of the drift velocity at different densities above $E_{\mathrm{bd}}$. The noise determinations of breakdown are more clear cut, they are added in Fig. 2(a) as black squares, in qualitative agreement with the velocity determination [47]. Focusing on the median values corresponding to $N=5$ (out of the experimental window $N=1 \rightarrow 10$ ), the noise data can be accurately fitted by the Zener tunneling limit $v_{c}=E_{c} / B=\omega_{c} l_{B} / \sqrt{N}$ (black dashed line) as shown in Fig. 2(a). The strong effect of LL quantization on noise is evidenced in Fig. 2(b), which gathers data of Fig. 1 taken in the doped regime $\left(V_{g}=-3 \mathrm{~V}\right)$, where noise is expressed in terms of a noise temperature $k_{B} T_{e}=S_{I} / 4 G_{\mathrm{sat}}$. $G_{\mathrm{sat}}$ being constant, this representation conveniently interpolates between quantum shot noise at $E \gtrsim E_{\mathrm{bd}}$ and thermal noise at $E \gg E_{\mathrm{bd}}$. The figure captures (i) the suppression of shot noise below $E_{\mathrm{bd}}$, (ii) the proliferation of inter-LL excitations above $E_{\mathrm{bd}}$, with $k_{B} T_{e} \sim e V_{\mathrm{ds}} / 2$, and (iii) their relaxation at ultimate bias. As seen in Fig. 2(c), the noise data themselves obey a QHE scaling where the $E$ field is scaled to the maximum Zener field, $\tilde{E}=E / E_{c}(N=1)$, and the noise temperature to the
Landau gap. One retrieves the Zener-like critical field with $\tilde{E}_{\text {bd }} \simeq 1 / \sqrt{5}$ in agreement with the theoretical fit in Fig. 2(a). In this plot, we have added $V_{g}=0$ data showing that $\tilde{E}_{\text {bd }} \rightarrow 1$ at neutrality as expected. The above QHE scaling supports the fundamental physics origin of shot noise and highlights the fact that the Fermi sea is sprayed over a very large number of LLs [ $\gtrsim 100$ in Fig. 2(c)] under the combined effects of LL quantization and QHE breakdown, and that noise itself scales like the cyclotron energy with $S_{I}\left(k_{F}, B, E\right) \propto \hbar \omega_{c} \times f\left(k_{F} l_{B}, \tilde{E}\right)$ at $\left(E_{\mathrm{bd}} \lesssim E \lesssim 2 E_{\mathrm{bd}}\right)$.

To gain a deeper insight into the breakdown mechanism, we compare in Fig. 3(a) the noise current with the backscattering current defined as the deviation from ballistic Hall transport, $I_{\mathrm{bs}}=G_{H} V_{\mathrm{ds}}-I_{\mathrm{ds}}$. At $E \gtrsim E_{\mathrm{bd}}$ the backscattering current identifies with the inter-LL tunneling current which is drained back to the source via a dense array of counterpropagating edge states. Both $S_{I}$ and $I_{\mathrm{bs}}$ vanish in the QHE regime $E<E_{\mathrm{bd}}$ and grow exponentially at $E \sim E_{\mathrm{bd}}$. As $S_{I}\left(V_{g}\right) \propto I_{\mathrm{bs}}\left(V_{g}\right)$, we can define an $E$-field and doping independent backscattering Fano factor $\mathcal{F}(B)=S_{I} / 2 e I_{\mathrm{bs}}$. At $B=3 \mathrm{~T}$ the large $\mathcal{F} \simeq 7.5$ in Fig. 3(a) points to a collective mechanism and rules out the single electron Zener interpretation. The inset of Fig. 3(a) shows the linear dependence $\mathcal{F}(B) \propto B$ which supports the QHE scaling of noise in Fig. 2(c). It introduces a constant energy scale, $\varepsilon_{c}=\hbar \omega_{c} / \mathcal{F} \simeq 1.4 \mathrm{meV}$, the origin of which remains to be clarified.

From a theoretical point of view, single-electron Zener tunneling is also excluded based on the necessary conservation of the wave vector in the direction perpendicular to the electric field, and one would need to appeal to impurities that break translation invariance or to electron-electron interactions. The latter give rise to collective excitations such as magnetoexcitons [28] with a dispersion 

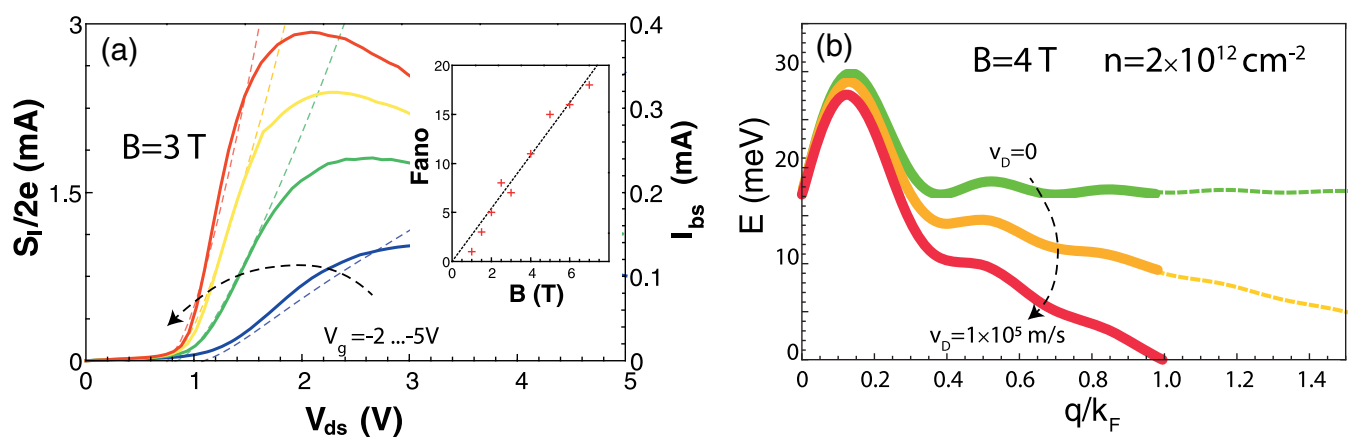

FIG. 3. Super-Poissonian inter-Landau level tunneling and a sketch of the magnetoexciton instability scenario of QHE breakdown. (a) Shot noise (solid lines, left axis) and backscattering current $I_{\mathrm{bs}}=G_{H} V_{\mathrm{ds}}-I_{\mathrm{ds}}$ (dashed lines, right axis) at $B=3 \mathrm{~T}$ for $V_{g}=$ $-2 \rightarrow-5 \mathrm{~V}$ (blue, green, orange, red) obey a similar bias dependence at breakdown yielding a doping independent Fano factor $\mathcal{F}=S_{I} / 2 e I_{\mathrm{bs}} \simeq 7.5$. Inset $\mathcal{F}(B) \simeq\left(2.7 T^{-1}\right) B \approx \hbar \omega_{c} / \varepsilon_{c}$ obeys a linear law with $\varepsilon_{c}=1.4 \mathrm{meV}$. (b) ME spectrum according to Eq. (1), at rest $\left(v_{D}=0\right)$, for a subcritical $\left(v_{D}=0.5 \times 10^{5} \mathrm{~m} / \mathrm{s}\right)$ and the critical $\left(v_{D}=1 \times 10^{5} \mathrm{~m} / \mathrm{s}\right)$ drift velocities. For clarity we have chosen a large $r_{s} \simeq 4, B=4 \mathrm{~T}$ and $|n|=2 \times 10^{12} \mathrm{~cm}^{12}(N=5)$, corresponding to the $V_{g}=-3 \mathrm{~V}$ median working point in (Fig. SM1 [33]). The line thickness illustrates the spectral weight which, according to Ref. [48], vanishes at $q \sim k_{F}$. The Landau critical velocity $v_{c}=1 \times 10^{5} \mathrm{~m} / \mathrm{s}$ is estimated by the vanishing of the ME gap at $q / k_{F} \sim 1$. It agrees with the noise determination of breakdown, $v_{\mathrm{bd}}=1.1 \times 10^{5} \mathrm{~m} / \mathrm{s}[B=4 \mathrm{~T}$ square in Fig. 2(a) $]$.

$\omega_{\mathrm{ME}}^{E}(q) \simeq \omega_{c}\left(1+\frac{r_{s}}{2 \pi} \frac{k_{F}}{q}\left[J_{1}\left(2 N q / k_{F}\right)\right]^{2}\right)-\mathbf{v}_{D} \cdot \mathbf{q}$,

in terms of the Bessel function $J_{1}(x)$ [33]. Here, the dopingdependent Seitz radius, $r_{s}=1 / a_{B}^{*} k_{F} \simeq 0.5 \ldots 1$, is given by the effective Bohr radius $a_{B}^{*}=0.5 \AA \times\left(m_{e} / m^{*}\right) \epsilon$, with $\epsilon=3.2$ for $\mathrm{BN}$. While magnetoexcitons are always gapped $\left[\omega_{\mathrm{ME}}^{E=0}(q) \geq \omega_{c}\right]$ in the absence of an electric field $(E=0)$, the latter tilts the dispersion by $-\mathbf{v}_{D} \cdot \mathbf{q}$. One immediately notices that the system becomes unstable towards proliferation of MEs because parts of the dispersion become negative at large wave vectors. However, in order to have a true instability, the excitation must have a nonzero spectral weight at the wave vectors where the dispersion vanishes. The spectral weight of the MEs is restricted to a wave-vector range $1 / \sqrt{2 N} l_{B} \leq q \leq 2 \sqrt{2 N} / l_{B}=2 k_{F}[38,39,48]$, and the instability thus occurs when $\omega_{\mathrm{ME}}^{E}\left(q \simeq 2 \sqrt{2 N} / l_{B}\right) \simeq$ $\omega_{c} \simeq 2 v_{D} \sqrt{2 N} / l_{B}$. This yields the above-mentioned scaling law $v_{c}=E_{c} / B \sim \omega_{c} l_{B} / \sqrt{N}$ for the critical velocity, and establishes the equivalence of the Zener and ME instability critical fields.

Figure 3(b) is a sketch of the low energy ME spectrum $\omega_{\mathrm{ME}}(q)-v_{D} q$ in increasing drift velocities $v_{D}=0,0.5$, and $1 \times 10^{5} \mathrm{~m} / \mathrm{s}$ for our typical experimental parameters $|n|=2 \times 10^{12} \mathrm{~cm}^{-2}\left(V_{g}=-3 \mathrm{~V}\right), B=4 \mathrm{~T}(N=5)$. Line thickness sketches ME's spectral weight, which vanishes for $q \gtrsim k_{F}$ as mentioned above. The ME instability occurs at a Landau critical velocity $v_{L} \sim \omega_{c} / k_{F} \simeq 1 \times 10^{5} \mathrm{~m} / \mathrm{s}$ in agreement with the experimental (4 T) value in Fig. 2(a), where $v_{\mathrm{bd}}=1.1 \times 10^{5} \mathrm{~m} / \mathrm{s}$.

We conclude from the above analysis that the singleelectron Zener tunneling and the collective ME instability mechanisms cannot be distinguished by their critical velocity, and that an independent diagnosis of their excitation yield is needed, such as the current noise in Figs. 1(a)-1(h). A first evidence is the large Fano factor observed at $E \gtrsim E_{\mathrm{bd}}$. In contrast with the Zener mechanism, the proliferation of inter-LL collective excitations is naturally explained by the ME instability. However, a quantitative determination of the number $N_{\text {bunch }}=\mathcal{F} \lesssim 20$ of excitation bunches remains beyond the scope of the present argument. In the Supplemental Material [33] we propose a tentative explanation that estimates a $N_{\text {bunch }}=\mathcal{F} \sim \hbar \omega_{c} / \varepsilon_{c}$ from the interedge charging energy $\varepsilon_{c} \sim e^{2} /(\epsilon W) \simeq 2 \mathrm{meV}$. A second evidence of the collective nature of breakdown comes from the current noise peak itself at $\mathcal{E} \gtrsim 2 \mathcal{E}_{\text {bd }}$. Its large amplitude, $S_{I} / 2 e \sim I_{\mathrm{ds}}$ in Figs. 1(a)-1(h) or $k_{B} T_{N}=$ $S_{I} / 4 G_{\text {sat }} \sim 100 \hbar \omega_{c} \sim e V_{\mathrm{ds}} / 2$ in Figs. 2(b), 2(c) suggests an alteration of occupation numbers in the full LL spectrum consistently with a ME instability. By contrast a Zener mechanism, which affects mostly LLs at Fermi energy leaving deep LLs unaffected, should lead to $S_{I} / 2 e<I_{\mathrm{ds}}$. It is further supported by the hint of a QHE-metal transition at very high electric fields $\left(E \gg E_{\mathrm{bd}}\right)$, characterized by a constant differential conductance $G_{\mathrm{ds}} \rightarrow G_{\mathrm{sat}} \simeq 0.6 \mathrm{mS}$ in Figs. 1(a)-1(h) and a decrease of thermal noise $S_{I}(B, E) \rightarrow$ $S_{I}(0, E)$ in Fig. 2(c). This trend is a natural consequence of the field-induced melting of LLs. Remarkably, it is accompanied by the ignition of hyperbolic substrate-phonon cooling, an efficient mechanism recently demonstrated using the same sample [37]. The suppression of phonon relaxation in quantizing fields, also reported in magneto-optic experiments [49], and its restoring upon LL merging, are experimental illustrations of the importance of the plane-wave nature of electrons in electron-phonon coupling.

In conclusion, the old problem of quantum Hall breakdown has been revisited in the light of high-frequency shot-noise measurements performed in high-mobility 
bilayer graphene. A new mechanism has been proposed that involves an electric-field driven bulk magnetoexciton instability. It explains the observed critical velocity and noise phenomenology as well as their scaling with the Zener field and the Landau energy. The mechanism makes a bridge between quantum-Hall and superfluid breakdowns by introducing a Landau critical drift velocity to the quantum Hall insulator state. This new approach will stimulate further theoretical and experimental works, aiming at modeling the $E$-field-induced QHE to metal transition or checking its universality, e.g., in single-layer graphene where Lorentzian invariance substitutes Galilean invariance, in ultraclean semiconducting heretostructures, or in the fractional quantum Hall effect.

The research leading to these results has received partial funding from the European Union "Horizon 2020" research and innovation programme under Grant Agreement No. 785219 "Graphene Core," from the ANR14-CE08-018-05 "GoBN." G. Z. acknowledges the financial support from the National Basic Research Program of China (973 Program) under Grant No. 2013CB934500, the National Science Foundation of China (NSFC) under Grant No. 61325021. A. B. acknowledges the financial support from the Foundation Cellex, the Centres de Recerca de Catalunya (CERCA) programme, Severo Ochoa (SEV-2015-0522) and Fondo Europeo de Desarollo Regional (FEDER).

*bernard.placais@1pa.ens.fr

[1] K. von Klitzing, G. Dorda, and M. Pepper, New Method for High-Accuracy Determination of the Fine-Structure Constant based on Quantized Hall Resistance, Phys. Rev. Lett. 45, 494 (1980).

[2] A. Tzalenchuk, S. Lara-Avila, A. Kalaboukhov, S. Paolillo, M. Syvajarvi, R. Yakimova, O. Kazakova, T. J. B. M. Janssen, V. Fal'ko, and S. Kubatkin, Towards a quantum resistance standard based on epitaxial graphene, Nat. Nanotechnol. 5, 186 (2010).

[3] R. Ribeiro-Palau, F. Lafont, J. Brun-Picard, D. Kazazis, A. Michon, F. Cheynis, O. Couturaud, C. Consejo, B. Jouault, W. Poirier, and F. Schopfer, Quantum Hall resistance standard in graphene devices under relaxed experimental conditions, Nat. Nanotechnol. 10, 965 (2015).

[4] E. Bocquillon, V. Freulon, F. D. Parmentier, J-M. Berroir, B. Plaçais, C. Wahl, J. Rech, T. Jonckheere, T. Martin, C. Grenier, D. Ferraro, P. Degiovanni, and G. Fève, Electron quantum optics in ballistic chiral conductors, Ann. Phys. (Berlin) 526, 1 (2014).

[5] P. Streda and K. von Klitzing, Critical non-dissipative current of the quantum Hall regime, J. Phys. C 17, L483 (1984).

[6] M.E. Cage, R. F. Dziuba, B.F. Field, E. R. Williams, S. M. Girvin, A. C. Gossard, D. C. Tsui, and R. J. Wagner, Dissipation and Dynamic Nonlinear Behavior in the Quantum Hall Regime, Phys. Rev. Lett. 51, 1374 (1983).
[7] L. Bliek, E. Braun, G. Hein, V. Kose, J. Niemeyer, G. Weimann, and W. Schlapp, Critical current density for the dissipationless quantum Hall effect, Semicond. Sci. Technol. 1, 110 (1986).

[8] L. Eaves and F. W. Sheard, Size-dependent quantised breakdown of the dissipationless quantum Hall effect in narrow channels, Semicond. Sci. Technol. 1, 346 (1986).

[9] I. A. Dmitriev, A. D. Mirlin, D. G. Polyakov, and M. A. Zudov, Nonequilibrium phenomena in high Landau levels, Rev. Mod. Phys. 84, 1709 (2012).

[10] C. Chaubet and F. Geniet, Nonequilibrium occupation of Landau levels and universal critical field in the quantumHall-effect breakdown, Phys. Rev. B 58, 13015 (1998).

[11] K. Chida, T. Arakawa, S. Matsuo, Y. Nishihara, T. Tanaka, D. Chiba, T. Ono, T. Hata, K. Kobayashi, and T. Machida, Nonequilibrium occupation of Landau levels and universal critical field in the quantum-Hall-effect breakdown, Phys. Rev. B 87, 155313 (2013).

[12] M.-Y. Li, T. Nakajima, K.-T. Lin, C. C. Chi, J. C. Chen, and S. Komiyama, Transition dynamics in the electrical breakdown of the quantum Hall effect, Phys. Rev. B 85, 245315 (2012).

[13] K. Panos, R. R. Gerhardts, J. Weis, and K. von Klitzing, Current distribution and Hall potential landscape towards breakdown of the quantum Hall effect: A scanning force microscopy investigation, New J. Phys. 16, 113071 (2014).

[14] B. E. Kane, D. C. Tsui, and G. Weimann, Evidence of Inter-Landau-Level Tunneling in the Integral Quantum Hall Effect, Phys. Rev. Lett. 61, 1123 (1988).

[15] I. I. Kaya, G. Nachtwei, K. von Klitzing, and K. Eberl, Spatially resolved monitoring of the evolution of the breakdown of the quantum Hall effect: Direct observation of inter-Landau-level tunneling, Europhys. Lett. 46, 62 (1999).

[16] J. A. Alexander-Webber, A. M. R. Baker, P. D. Buckle, T. Ashley, and R. J. Nicholas, High-current breakdown of the quantum Hall effect and electron heating in InSb/AlInSb, Phys. Rev. B 86, 045404 (2012).

[17] J. Guignard, D. Leprat, D. C. Glattli, F. Schopfer, and W. Poirier, Quantum Hall effect in exfoliated graphene affected by charged impurities: Metrological measurements, Phys. Rev. B 85, 165420 (2012).

[18] J. A. Alexander-Webber, A. M. R. Baker, T. J. B. M. Janssen, A. Tzalenchuk, S. Lara-Avila, S. Kubatkin, R. Yakimova, B. A. Piot, D. K. Maude, and R. J. Nicholas, Phase Space for the Breakdown of the Quantum Hall Effect in Epitaxial Graphene, Phys. Rev. Lett. 111, 096601 (2013).

[19] A. M. R. Baker, J. A. Alexander-Webber, T. Altebaeumer, and R. J. Nicholas, Energy relaxation for hot Dirac fermions in graphene and breakdown of the quantum Hall effect, Phys. Rev. B 85, 115403 (2012).

[20] S. Tian, P. Wang, X. Liu, J. Zhu, H. Fu, T. Taniguchi, K. Watanabe, J.-H. Chen, and X. Lin, Nonlinear transport of graphene in the quantum Hall regime, 2D Mater. 4, 015003 (2017)

[21] K. Chida, T. Hata, T. Arakawa, S. Matsuo, Y. Nishihara, T. Tanaka, T. Ono, and K. Kobayashi, Avalanche electron bunching in a Corbino disk in the quantum Hall effect breakdown regime, Phys. Rev. B 89, 235318 (2014).

[22] T. Hata, T. Arakawa, K. Chida, S. Matsuo, and K. Kobayashi, Giant Fano factor and bistability in a Corbino 
disk in the quantum Hall effect breakdown regime, J. Phys. Condens. Matter 28, 055801 (2016).

[23] A. Laitinen, M. Kumar, T. Elo, Y. Liu, T. S. Abhilash, and P. J. Hakonen, Breakdown of zero-energy quantum Hall state in Graphene in the light of current fluctuations and shot noise, J. Low Temp. Phys. 191, 272 (2018).

[24] A. Laitinen, M. Kumar, P. J. Hakonen, and E. B. Sonin, Gyrotropic Zener tunneling and nonlinear IV curves in the zero-energy Landau level of graphene in a strong magnetic field, Sci. Rep. 8, 594 (2018).

[25] S. Komiyama, T. Takamasu, S. Hiyamizu, and S. Sasa, Breakdown of the quantum Hall effect due to electron heating, Solid State Commun. 54, 479 (1985).

[26] G. Nachtwei, Breakdown of the quantum Hall effect, Physica (Amsterdam) 4E, 79 (1999).

[27] S. Komiyama and Y. Kawaguchi, Heat instability of quantum Hall conductors Phys. Rev. B 61, 2014 (2000).

[28] C. Kallin and B. I. Halperin, Excitations from a filled Landau level in the two-dimensional electron gas, Phys. Rev. B 30, 5655 (1984); Many-body effects on the cyclotron resonance in a two-dimensional electron gas, Phys. Rev. B 31, 3635 (1985).

[29] S. M. Girvin, A. H. MacDonald, and P. M. Platzman, Magneto-roton theory of collective excitations in the fractional quantum Hall effect, Phys. Rev. B 33, 2481 (1986).

[30] L. Landau, Theory of the Superfluidity of Helium II, Phys. Rev. 60, 356 (1941).

[31] D. I. Bradley, S. N. Fisher, A. M. Guénault, R. P. Haley, C. R. Lawson, G. R. Pickett, R. Schanen, M. Skyba, V. Tsepelin, and D. E. Zmeev, Breaking the superfluid speed limit in a fermionic condensate, Nat. Phys. 12, 1017 (2016).

[32] L. Chomaz, R. M. W. van Bijnen, D. Petter, G. Faraoni, S. Baier, J. H. Becher, M. J. Mark, F. Wächtler, L. Santos, and F. Ferlaino, Observation of roton mode population in a dipolar quantum gas, Nat. Phys. 14, 442 (2018).

[33] See Supplemental Material at http://link.aps.org/ supplemental/10.1103/PhysRevLett.121.136804 for a derivation of the magnetoexciton dispersion. It contains Refs. [28,34-39].

[34] M. O. Goerbig, Quantum Hall Effects, in Ultracold Gases and Quantum Information, Proceedings of the Les Houches Summer School 2009, edited by C. Miniatura et al. (Oxford University Press, Oxford, 2009), arXiv:0909.1998.

[35] G. F. Giuliani and G. Vigniale, Quantum Theory of the Electron Liquid (Cambridge University Press, Cambridge, England, 2005).

[36] J. Koch and F. von Oppen, Franck-Condon Blockade and Giant Fano Factors in Transport through Single Molecules, Phys. Rev. Lett. 94, 206804 (2005).

[37] W. Yang, S. Berthou, X. Lu, Q. Wilmart, A. Denis, M. Rosticher, T. Taniguchi, K. Watanabe, G. Fève, J.-M. Berroir,
G. Zhang, C. Voisin, E. Baudin, and B. Plaçais, A graphene Zener-Klein transistor cooled by a hyperbolic substrate, Nat. Nanotechnol. 13, 47 (2018).

[38] R. Roldán, M. O. Goerbig, and J.-N. Fuchs, The magnetic field particle-hole excitation spectrum in doped graphene and in a standard two-dimensional electron gas, Semicond. Sci. Technol. 25, 034005 (2010).

[39] M. O. Goerbig, Electronic properties of graphene in a strong magnetic field, Rev. Mod. Phys. 83, 1193 (2011).

[40] A. M. Martin, K. A. Benedict, F. W. Sheard, and L. Eaves, Model for the Voltage Steps in the Breakdown of the Integer Quantum Hall Effect, Phys. Rev. Lett. 91, 126803 (2003).

[41] L. Eaves, S. T. Stoddart, R. Wirtz, A. C. Neumann, B. L. Gallagher, P. C. Main, and M. Henini, Quantum Hall effect breakdown in two-dimensional hole gases, Physica (Amsterdam) 6E, 136 (2000).

[42] Actually a residual current noise is observed in the ballistic $\mathrm{QH}$ regime, which is hardly visible in Figs. 1(a)-1(h) and 2(c). It corresponds to a noise temperature $k_{B} T_{N}=$ $S_{I} / 4 G_{d s} \lesssim 0.02 e V_{d s}$ that is one order of magnitude smaller than the hot electron limit $k_{B} T_{N}=\sqrt{3} / 8 e V_{d s}$ for homogeneous Joule heating [37]. We attribute it to the local contact heating associated with the Joule power release in the source and drain.

[43] Note that in a two-terminal device the electric field rotates from transverse to longitudinal as transport turns from the dissipationless Hall regime to the dissipative Ohmic regime.

[44] A. C. Betz, F. Vialla, D. Brunel, C. Voisin, M. Picher, A. Cavanna, A. Madouri, G. Fève, J.-M. Berroir, B. Plaçais, and E. Pallecchi, Hot Electron Cooling by Acoustic Phonons in Graphene, Phys. Rev. Lett. 109, 056805 (2012).

[45] A. C. Betz, S. H. Jhang, E. Pallecchi, R. Ferreira, G. Fève, J.-M. Berroir, and B. Plaçais, Supercollision cooling in undoped graphene, Nat. Phys. 9, 109 (2013).

[46] A. Laitinen, M. Kumar, M. Oksanen, B. Plaçais, P. Virtanen, and P. J. Hakonen, Coupling between electrons and optical phonons in suspended bilayer graphene, Phys. Rev. B 91, 121414(R) (2015).

[47] Note that bunching quality depends crucially on the accuracy of the drain gating compensation as explained in Ref. [37].

[48] R. Roldán, J.-N. Fuchs, and M. O. Goerbig, Spin-flip excitations, spin waves, and magnetoexcitons in graphene Landau levels at integer filling factors, Phys. Rev. B 82, 205418 (2010).

[49] F. Wendler, M. Mittendorff, J. C. König-Otto, S. Brem, C. Berger, W. A. de Heer, R. Böttger, H. Schneider, M. Helm, S. Winnerl, and E. Malic, Symmetry-Breaking Supercollisions in Landau-Quantized Graphene, Phys. Rev. Lett. 119, 067405 (2017). 